\title{
Identification of Industrial Robot Parameters for Advanced Model-Based Controllers Design *
}

\author{
Basilio Bona and Aldo Curatella \\ Dipartimento di Automatica e Informatica \\ Politecnico di Torino \\ Corso Duca degli Abruzzi 24, 10129 Torino, Italy \\ basilio. bona@polito. it \\ cataldo. curatella@polito. it
}

\begin{abstract}
This paper presents the identification process aimed at estimating the model parameters of a COMAU Smart S2 industrial robot for controller design purposes. The principal challenges are given by the presence of a controller-in-the-loop, and the absence of sensors to measure joint accelerations and velocities. A method valid in this context has been applied, and suitable trajectories were generated in order to avoid to excite the unmodelled plant dynamic while allowing to compute analytically the joint velocities and accelerations. This method was then applied to a real 6 degrees of freedom (DoF) industrial robot, estimating and using its parameters to design new improved model-based controllers.
\end{abstract}

Keywords: Identification, base parameters estimation, closed-loop identification, robot dynamics.

\section{Introduction}

Robots find intensive applications in factories to improve product quality and safety, while reducing costs and processing time. To improve performances it is often necessary to design advanced model-based controllers that require an accurate knowledge of the robot model parameters. Since the robot producers very rarely provide this information, users need to establish a reliable procedure allowing to generate the best model in the frequency range of interest.

The robot model depends on the inertia, mass and center of mass of each link, but the number of parameters increases with the number of DoF, requiring more computational work.

To avoid this problem, a set of parameters, named Base Parameters, should be used [2]-[6]; they are the minimum number of inertial parameters able to correctly describe the dynamic behaviour of the robot.

Many identification methods exist for unknown open loop systems [7], but several of them fail when used for closed loop systems, as in the robot case; indeed a robot cannot operate without an active controller, for safety or production reasons, so one of the methods for closed loop system identification [7]-[11] shall be used instead; the socalled Projection Method (PM) has been chosen since it does not rely on particular assumptions about the controller structure.

Velocity and acceleration measurements are necessary in the identification process, but often the relevant sensors are not present for cost reasons. To avoid numerical derivation, a Finite Fourier series was used to generate reference trajectories; this allow to average the data, improve the signal-to-noise ratio, compute statistical characteristic of noise measurements, and define the bandwidth of the exciting trajectory.

\footnotetext{
*This work was partially supported by MIUR 2002 PRIN "Matrics" funding grant.
} 
Section 2 introduces the (rigid) robot model and its parameters, and reviews possible identification methods, while in Section 3 the results obtained implementing the PM method on a real COMAU Smart-3 S2 industrial robot will be presented and discussed. Conclusions are drawn in Section 4.

\section{Robot Model and Parameters}

For the identification process, the assumed model is based on open-chain $n$-links physical model, with the following additional hypothesis:

- rigid links and joints are assumed, i.e. elastic potential energy storage elements are not considered;

- joint gearboxes are ideal, i.e. $100 \%$ efficient, without dead bands, etc.

- friction is modelled as the sum of viscous and Coulomb friction only, i.e. no stiction is considered.

For each link $i=1, \ldots, n$, Lagrange equations are applied $[1,2]$ :

$$
\frac{d}{d t}\left(\frac{\partial \mathcal{L}}{\partial \dot{q}_{i}}\right)-\frac{\partial \mathcal{L}}{\partial q_{i}}=\tau_{i}-f_{i}^{f} \quad \forall i=1, \ldots, n
$$

where $n$ is the number of joints, $\mathcal{L}$ is the Lagrange Energy Function

$$
\mathcal{L}(\boldsymbol{q}, \dot{\boldsymbol{q}})=\mathcal{C}(\boldsymbol{q}, \dot{\boldsymbol{q}})-\mathcal{P}(\boldsymbol{q})
$$

where $\mathcal{C}$ and $\mathcal{P}$, are respectively, the kinetic coenergy and the potential energy, $\boldsymbol{q} \in \mathbb{R}^{n}$ is the joint position vector, $\dot{\boldsymbol{q}} \in \mathbb{R}^{n}$ the joint velocity vector, $q_{i}$ and $\dot{q}_{i}$ the $i$-th joint position and velocity respectively, while $f_{i}^{f}$ is the friction force acting on the $i$-th joint, modelled as:

$$
f_{i}^{f}\left(\dot{q}_{i}\right)= \begin{cases}\beta_{i}^{v+} \dot{q}_{i}+\beta_{i}^{c+} & \dot{q}_{i}>0 \\ 0 & \dot{q}_{i}=0 \\ \beta_{i}^{v-} \dot{q}_{i}+\beta_{i}^{c-} & \dot{q}_{i}<0\end{cases}
$$

The link parameters to be estimated are the inertial parameters (mass $m_{i}$, inertia tensor $\boldsymbol{\Gamma}_{i}$ and center of mass $\boldsymbol{c}_{i}$ expressed in local coordinate frames assigned to individual axes) and friction parameters $\left(\beta_{i}^{v+}, \beta_{i}^{v-}, \beta_{i}^{c+}, \beta_{i}^{c-}\right)$.

\subsection{Base Parameters}

Ten inertial parameters must be estimated for every link and the numerical complexity of the procedure increases with the robot's DoF. Fortunately, not all of them influence the dynamic behaviour and others may be regrouped as linear combinations; those that influence dynamics are called Base Parameters and are the only one to be estimated [3]-[6].

Kinetic coenergy $\mathcal{C}$ and potential energy $\mathcal{P}$ can be written as linear combinations of inertial parameters, so that if the following conditions hold for some parameter:

$$
D \mathcal{C}_{j}=0 \text { and } \quad d \mathcal{P}_{j}=0
$$

that parameter has no effect on the dynamic model and does not belong to the base parameter set. Inertial parameters satisfying (3) are relative to the axes near the base, and there are rules [3,4] to find these parameters without using $(3)$.

\subsection{Identification Method}

With the base parameters set we can write the model as a linear regression:

$$
\Phi(\boldsymbol{q}, \dot{\boldsymbol{q}}, \ddot{\boldsymbol{q}}) \boldsymbol{\theta}=\tau
$$

where $\boldsymbol{\theta}=\left[\boldsymbol{\theta}_{B}, \boldsymbol{\theta}_{f}\right]^{T}$ is the vector of parameters to be estimated, with $\boldsymbol{\theta}_{B}$ the base parameters vector and $\boldsymbol{\theta}_{f}$ the friction parameters vector. $\boldsymbol{\Phi}$ is the linear regressor which include dynamic and friction models of the robot, that depend on the joint positions, velocities and accelerations, while $\boldsymbol{\tau}$ is the command torque. 
There exist different methods [7] to estimated $\boldsymbol{\theta}$ in (4); all these methods are valid for open loop systems, but, since the robots operate in closed loop, some of these methods may fail when applied to them. Figure 1 represents a SISO closed-loop system, where $G$ is the system to be identified, $C$ the controller, $H$ models the noise dynamics, $e(t)$ is a white noise disturbance, $r(t)$ is the reference signal, $u(t)$ the torque command and $y(t)$ the measured position of the robot.

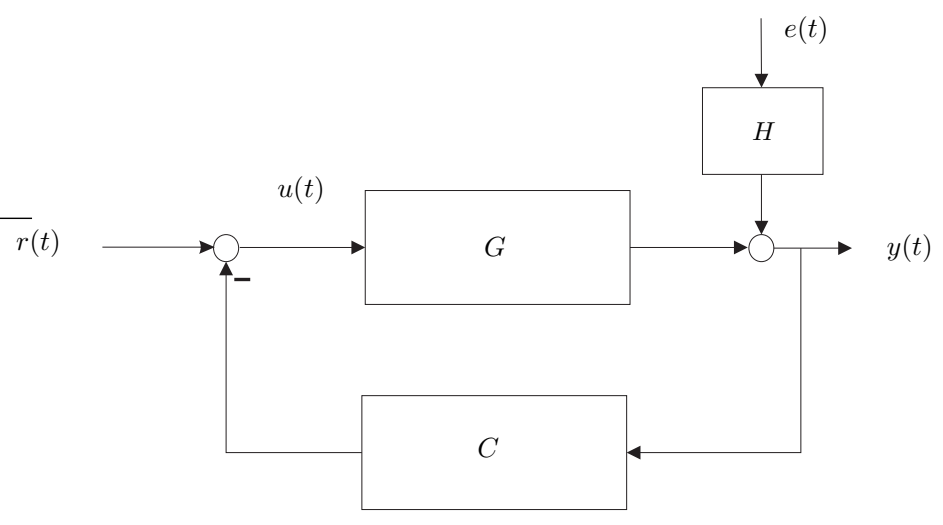

Fig. 1: SISO Closed-Loop System.

In this work, the Projection Method (PM) [8, 9] was used; it is a two-stage method (a particular case of joint input-output method), and operates as follows:

1. In the first stage, the sensitivity function $S$ is estimated using a non causal FIR (Finite Impulse Response) filter from the equation:

$$
\begin{aligned}
u(t) & =S(q, \boldsymbol{\theta}) r(t)+H_{2}(q, \boldsymbol{\theta}) e(t) \\
& =\sum_{k=-\mu_{1}}^{\mu_{2}} s_{k} r(t-k)+H_{2}(q, \boldsymbol{\theta}) e(t)
\end{aligned}
$$

then an estimate of the torque command is computed:

$$
\hat{u}(t)=\hat{S}(q, \boldsymbol{\theta}) r(t)=\sum_{k=-\mu_{1}}^{\mu_{2}} s_{k} r(t-k)
$$

where $\mu_{1}$ and $\mu_{2}$ are two integers chosen to avoid correlation between $\hat{u}(t)$ and $\tilde{u}(t)=u(t)-\hat{u}(t) ; q^{-k} r(t)=r(t-k)$ is the time-shift operator.

2. In the second stage, from (6) we can write:

$$
y(t)=G(q, \boldsymbol{\theta}) \hat{u}(t)+H_{1}(q, \boldsymbol{\theta}) e(t)
$$

and employ this equation to estimate the system transfer function $G$ with one of the open loop identification methods.

This method finds the best projection of the torque command $u(t)$ in the space defined by the reference $r(t)$, obtaining an estimate $\hat{u}(t)$ without the loop influence.

\subsubsection{Maximum Likelihood Estimation}

The second stage of PM requires a procedure to estimate the parameters in (7). A Maximum Likelihood Estimation Algorithm (MLE) [12] was used, estimating the value of $\boldsymbol{\theta}$ that maximizes the likelihood of the positions $\boldsymbol{q}(t)$ and torque $\boldsymbol{\tau}(t)$ measurements. 
Since noise on the measurements is always present, it has been assumed to be white gaussian with zero mean and variance $\boldsymbol{\sigma}^{2}$. The estimate $\hat{\boldsymbol{\theta}}_{M L}$ of $\boldsymbol{\theta}$ solves the constrained optimization problem:

$$
\begin{array}{r}
\min \frac{1}{2} \sum_{i, j=1}^{n}\left({ }^{i} \boldsymbol{x}-{ }^{j} \boldsymbol{\varphi}\right){ }^{T} \sigma_{i j}^{-2}\left({ }^{i} \boldsymbol{x}-{ }^{j} \boldsymbol{\varphi}\right) \\
\text { subject to } \\
M_{k}\left(\boldsymbol{\theta},{ }^{i} \boldsymbol{\varphi}\right)=0 \quad \forall i, k
\end{array}
$$

where ${ }^{i} \boldsymbol{\varphi}$ is the unknown true value of the $i$ th observation and ${ }^{i} \boldsymbol{x}$ is the measurement corrupted by noise ${ }^{i} \boldsymbol{x}={ }^{i} \boldsymbol{\varphi}+{ }^{i} \boldsymbol{\epsilon}$ $\forall i=1, \ldots n$, where $n$ is the observation number and $M_{k}$ is the dynamic model for the link $k$.

From (8) the estimate $\hat{\boldsymbol{\theta}}_{M L}$ of $\boldsymbol{\theta}$ follows:

$$
\hat{\boldsymbol{\theta}}_{M L}=\arg \min _{\boldsymbol{\theta}} \frac{1}{2} \sum_{i=1}^{n}{ }^{i} \boldsymbol{b}(\boldsymbol{\theta})^{T}{ }^{i} \boldsymbol{S}_{M L}(\boldsymbol{\theta})^{-2}{ }^{i} \boldsymbol{b}(\boldsymbol{\theta})
$$

where:

$$
\begin{aligned}
{ }^{i} \boldsymbol{b}(\boldsymbol{\theta}) & =-\left[\begin{array}{c}
M_{1}\left(\boldsymbol{\theta},{ }^{i} \boldsymbol{x}\right) \\
\vdots \\
M_{6}\left(\boldsymbol{\theta},{ }^{i} \boldsymbol{x}\right)
\end{array}\right] \\
{ }^{i} \boldsymbol{B}(\boldsymbol{\theta}) & =\left[\begin{array}{c}
\nabla_{{ }^{i} \boldsymbol{x}} M_{1}\left(\boldsymbol{\theta},{ }^{i} \boldsymbol{x}\right) \\
\vdots \\
\nabla_{{ }^{i} \boldsymbol{x}} M_{6}\left(\boldsymbol{\theta},{ }^{i} \boldsymbol{x}\right)
\end{array}\right] \\
{ }^{i} \boldsymbol{S}_{M L}(\boldsymbol{\theta})^{2} & ={ }^{i} \boldsymbol{B}(\boldsymbol{\theta}){ }^{i} \boldsymbol{\sigma}^{2}{ }^{i} \boldsymbol{B}(\boldsymbol{\theta})^{T}
\end{aligned}
$$

Equation (11) gives the covariance matrix of the model parameters estimated with the MLE.

\subsection{Generation of the Robot Trajectories}

Since the observation time is finite, aliasing errors are present; to avoid these leakage errors a periodic exciting trajectory was planned, repeating the measurements to obtain a white gaussian noise error, according to the Central Limit Theorem.

No information about joint velocities and accelerations is available; their numerical derivation causes noise amplification making them unusable. If the above quantities are analytic functions of the position, then they can be computed by analytical derivation; for this reason an exciting trajectory given by the following Finite Fourier series has been selected [13]:

$$
\begin{aligned}
& q_{i}(t)=\sum_{k=1}^{N_{i}}\left\{\frac{a_{i}(k)}{2 \pi f_{o} k} \sin \left(2 \pi f_{o} k t\right)-\right. \\
& \left.\frac{b_{i}(k)}{2 \pi f_{o} k} \cos \left(2 \pi f_{o} k t\right)\right\}+q_{i 0} \\
& \dot{q}_{i}(t)=\sum_{k=1}^{N_{i}}\left\{a_{i}(k) \cos \left(2 \pi f_{o} k t\right)+\right. \\
& \ddot{q}_{i}(t)=\sum_{k=1}^{\left.N_{i}(k) \sin \left(2 \pi f_{o} k t\right)\right\}}\left\{\begin{array}{c}
-\left(a_{i}(k) 2 \pi f_{o} k\right) \sin \left(2 \pi f_{o} k t\right)+ \\
\left.\left(b_{i}(k) 2 \pi f_{o} k\right) \cos \left(2 \pi f_{o} k\right)\right\}
\end{array}\right.
\end{aligned}
$$


where $f_{o}$ is the fundamental frequency and $N_{i}$ the harmonics number. The choice of $f_{o}$ and $N_{i}$ determines the bandwidth of the exciting trajectory so we can choose a specific bandwidth to avoid excitation of the elastic modes of the structure. Moreover, the reference signal should be persistently exciting with an order greater or equal to the model order to identify.

With this exciting trajectory only the $2 N_{i}+1$ parameters

$$
\boldsymbol{\delta}_{i}=\left[a_{i}(1), \ldots, a_{i}\left(N_{i}\right), b_{i}(1), \ldots, b_{i}\left(N_{i}\right), q_{i 0}\right]^{\top}
$$

$\forall i=1, \ldots, 6$ are to be computed. The vector $\boldsymbol{\delta}_{i}$ shall maximize the information of the observations; to do this [14] the Singular Value Decomposition (SVD) of the regressor $\boldsymbol{\Phi}$ was considered and its condition number $\kappa(\boldsymbol{\Phi})$ computed so that the optimal parametrization of the exciting trajectory is given by:

$$
\hat{\boldsymbol{\delta}}_{i}=\arg \min _{\boldsymbol{\delta}_{i}} \kappa(\boldsymbol{\Phi})
$$

subject to given position, velocity and acceleration constrains on each joint and end-effector constrains.

\section{Test Case: COMAU Smart-3 S2 Robot}

The approach presented was tested on a Robot COMAU Smart-3 S2 (see Figure 2), an anthropomorphic robot with $n=6 \mathrm{DoF}$; each joint has a gearbox with an high gear ratio that reduces the interaction among joints, so that a joint-independent model of the robot can be used.

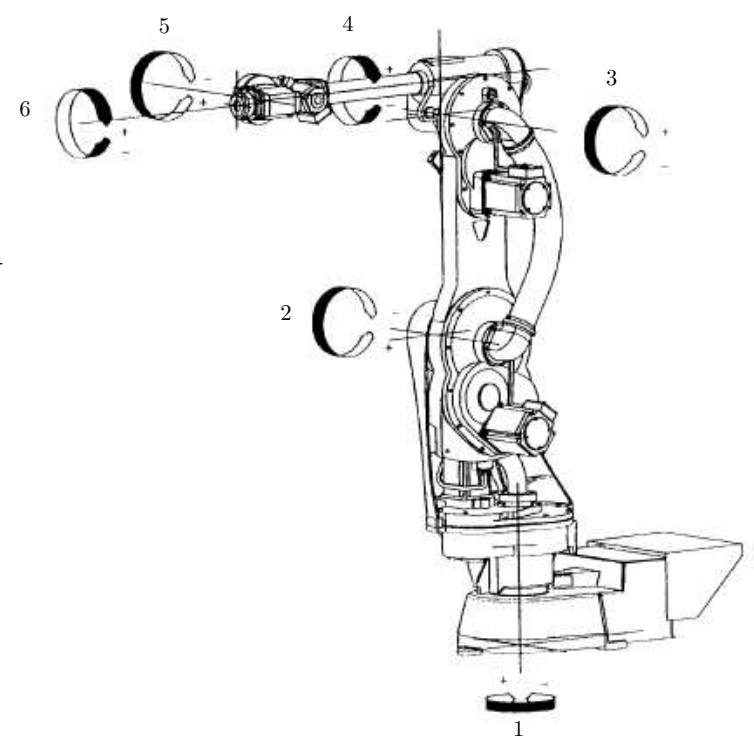

Fig. 2: Robot COMAU Smart-3 S2

One joint at at time was moved while the others were kept static, so that only its inertia and gravity effects are to be taken into account.

\subsection{Robot Model}

Each joint was modelled by the Lagrange equation (1):

$$
\ddot{q}_{i}(t)=-\frac{h_{i}\left(\boldsymbol{q}, \dot{q}_{i}\right)}{M_{i}}+\frac{\tau_{i}(t)}{M_{i}} \quad \forall i=1, \ldots, 6
$$

where $M_{i}$ is the mass matrix, the inertia of the $i$-th link, and $h_{i}\left(\boldsymbol{q}, \dot{q}_{i}\right)$ include gravity and friction effects. 


\subsection{Friction Model}

The unknown friction parameters are part of the linear regressor (4). So they have an important role in determining the parametrization of the trajectory (15), increasing the complexity and the computing time. To reduce them it is possible to estimate the friction parameters in (2) with specific observations and use them in the generation of the trajectory for parameters identification in (16).

\subsubsection{Gravity Compensation}

Before proceeding with the friction estimation, we should consider that gravity has remarkable effects on the robot behaviour, and that its effects must be compensated. Except for the second and third joint, it is possible to position the robot in order to have the other axes parallel to the gravity vector, compensating its effects.

If we model the link $i$ as an inverse pendulum with mass $M$ (mass of the links towards the end effector) on the tip, the gravity model is approximately sinusoidal:

$$
\tau_{g i}=k_{i} \sin \left(q_{i}+q_{i}^{\text {off }}\right)
$$

where $k_{i}$ is the amplitude of the torque gravity and $q_{i}^{\text {off }}$ models the offset position of the center of mass toward the center of the link.

To neglect inertia effects and have constant Coriolis, centripetal and friction effect, two slope trajectories, with the same velocity amplitude but opposite sign, were used. The results are reported in Figure 3, where we observe a sinusoidal torque and a constant offset due to the effects that depends linearly from the constant velocity of the trajectories. Then the gravity parameters was estimated, reported in Table 1; in Figure 3 (dashed line) the torque estimated using (17) was reported.
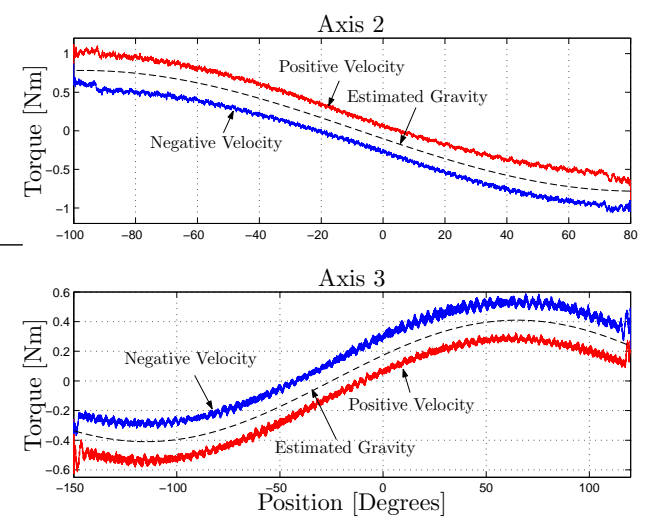

Fig. 3: Torque Measured and Gravity Torque Estimated

Tab. 1: Gravity Parameters Estimated

\begin{tabular}{|c|c|c|}
\hline Parameter & Axis 2 & Axis 3 \\
\hline \hline$k$ & $-0,7823 \mathrm{Nm}$ & $0,4097 \mathrm{Nm}$ \\
$q^{\text {off }}$ & $7,5^{\circ}$ & $25^{\circ}$ \\
\hline
\end{tabular}

\subsubsection{Friction Identification}

It is necessary to generate trajectories that enable to recover information about viscous and Coulomb friction, as modelled in (2). 
A Bell-Type position trajectory was used for all links; with this trajectory the inertia has opposite effects, since during acceleration it contrast the motion causing a boost of the torque and in deceleration it sustains the motion causing a reduction of the torque.

As expected, plotting ${ }^{1}$ the measurements for axis 2 in Figure 4, we can notice an hysteresis due to the link inertia and a bending of the plot due to the gravity effects. Compensating the hysteresis and the gravity, using the parameters from Table 1, we found a value of the torque to be associated only to the friction effects ${ }^{2}$. With the Least Square method we estimated the friction parameters: the results, reported in Table 2, were used to estimate the friction torque (dashed line in Figure 4).

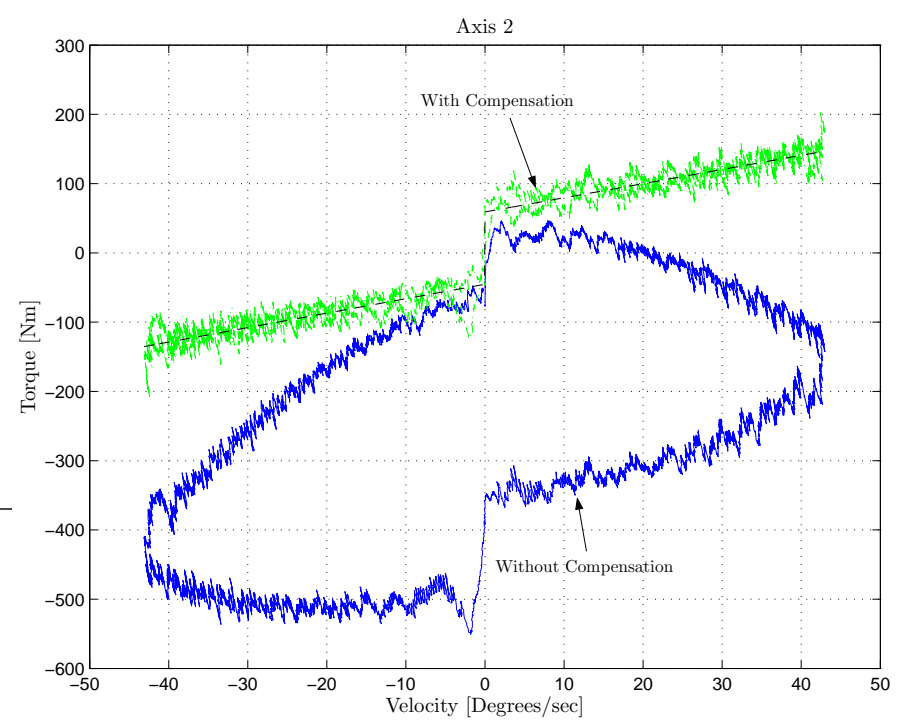

Fig. 4: Torque Measured for Gravity Compensation

Tab. 2: Friction Parameters Estimated

\begin{tabular}{|c|c|c|c|c|}
\hline Parameter & $\beta^{v+}$ & $\beta^{v-}$ & $\beta^{c+}$ & $\beta^{c-}$ \\
\hline \hline Axis 1 & 31,8757 & 26,0000 & 16,1376 & 15,0676 \\
Axis 2 & 36,6197 & 73,0530 & 65,4187 & 45,9598 \\
Axis 3 & 10,6964 & 11,5955 & 13,3809 & 11,3347 \\
Axis 4 & 3,0686 & 3,1806 & 2,0526 & 1,9685 \\
Axis 5 & 3,5672 & 3,2386 & 1,7545 & 1,7161 \\
Axis 6 & 1,8245 & 1,9985 & 2.4545 & 2.4161 \\
\hline
\end{tabular}

\subsection{Trajectory Generation}

The trajectory used in the experiment was based on the method presented in Section 2.3. Since the COMAU Smart-3 $\mathrm{S} 2$ robot has a resonance frequency between 3 and $20 \mathrm{~Hz}$ for the shoulder joints, and between 5 and $30 \mathrm{~Hz}$ for the wrist joints, the order of the joint model is 2 and the sampling frequency is $f_{s}=1 \mathrm{kHz}$, we choose a fundamental frequency of $f_{0}=0.1 \mathrm{~Hz}$ and $N_{i}=5$ harmonics with a bandwidth of $B_{w}=0.5 \mathrm{~Hz}$, which is considerably less than the minimum resonance frequency of the shoulder joints.

\footnotetext{
${ }^{1}$ For space reasons, only the results of one axis are reported.

2 Coriolis and centripetal effects are neglected in this discussion.
} 
Using the estimated friction parameters, a trajectory has been generated with the fmincon function of the Matlab Optimization Toolbox; the iteration process was stopped after 2000 iterations.

\subsection{Parameters Identification}

Every observation was performed 25 times, filtering the data with an 8-th order Chebyshev low pass filter, with cut-off frequency $f_{c}=80 \mathrm{~Hz}$ and resampled at $f_{s}=200 \mathrm{~Hz}$. From the averaged data the probability density function (PDF) of the measurement noise was computed and compared with a gaussian one (see Figure 5). From Figure 5 it results that the torque noise is greater than the position noise so that we can consider only the former.
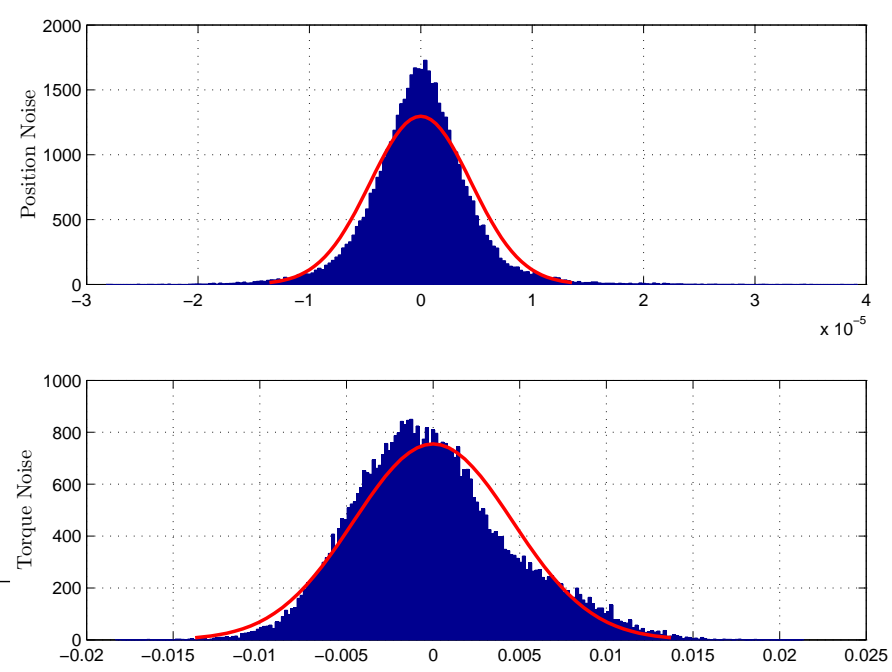

Fig. 5: Measurement Noise PDF for Axis 3

To correctly identify robot parameters, we should compensate friction effects using the friction parameters from Table 2 and obtaining a value of torque depending only on the robot dynamics (see Figure 6).

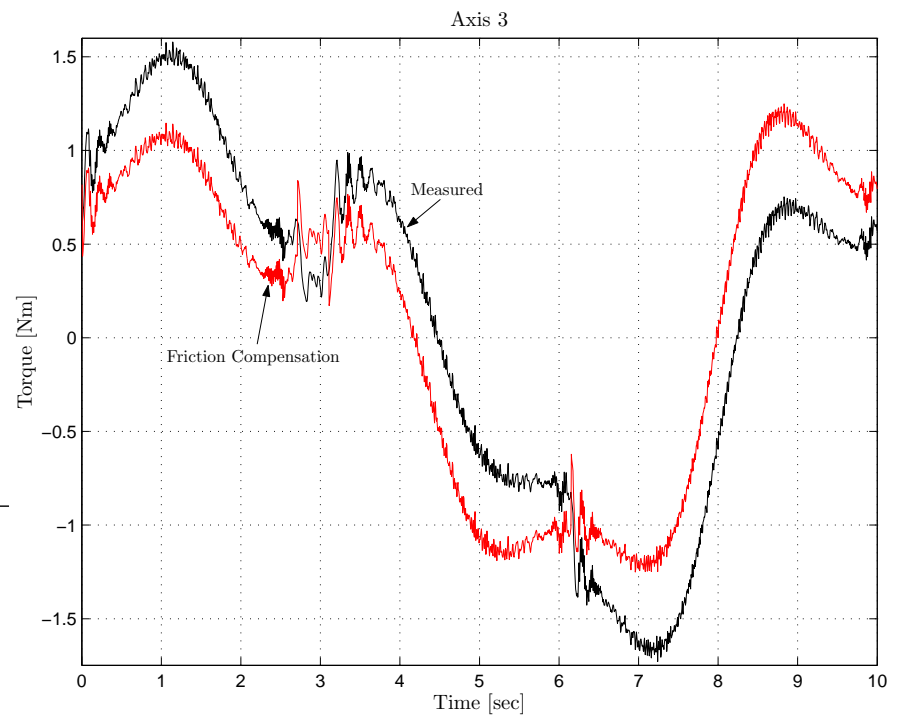

Fig. 6: Torque Measured for Friction Compensation 
In the first step of the PM procedure, the excitation spectrum was computed using the Fast Fourier Transform (FFT), that has spectrum lines only at $0.1 \mathrm{~Hz}$ and its multiples until $0.5 \mathrm{~Hz}$. Computing the actual torque spectrum, we find that there are some spectrum lines over $0.5 \mathrm{~Hz}$, apparently due to the feedback loop, that gives rise to a correlation between the noise measurements. The best estimate is obtained filtering the frequencies greater than the bandwidth (see Figure 7 ); a correlation coefficient between the estimate $\hat{\tau}_{3}$ and the estimation error $\tilde{\tau}_{3}=\tau_{3}-\hat{\tau}_{3}$ was computed as $\Phi_{\hat{\tau}_{3} \tilde{\tau}_{3}}=2.9238 \mathrm{e}^{-14}$ with no correlation between the two signals.

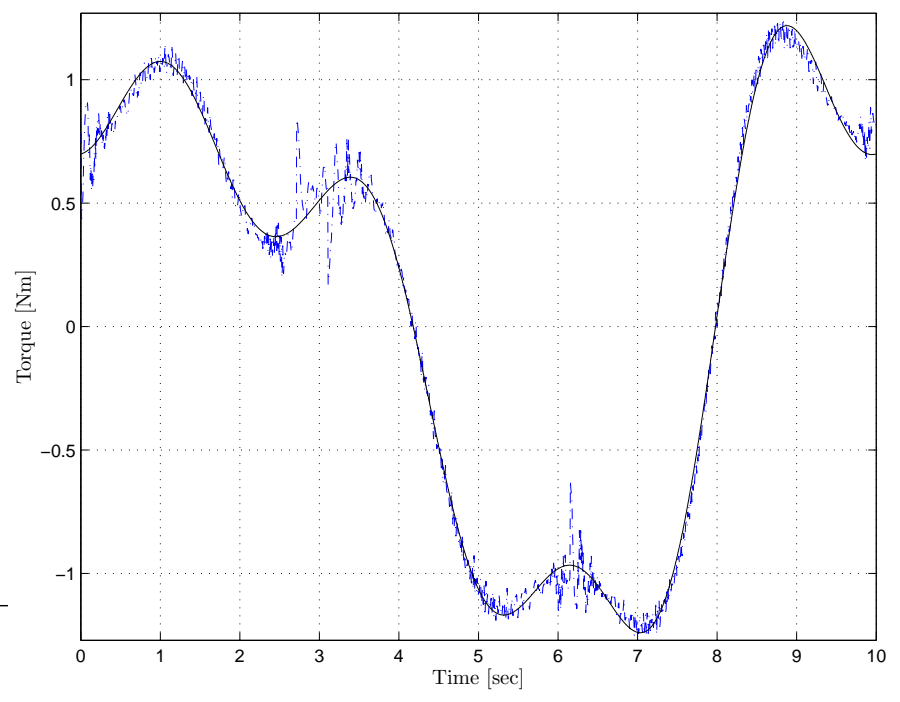

Fig. 7: Axis 3 Torque (dash-dotted: Measured, solid: Estimated)

In the second step, robot model parameters were estimated using estimated torque $\hat{\tau}_{i}$ with a MLE algorithm; shown in Table 3. For each link, the following quantities are reported: the parameter symbol with its unit of measure, the mean value and the standard deviation obtained in the identification process and the half amplitude of the $99.9 \%$ confidence interval, with $t_{\gamma}$ found using the Student distribution with $(n-1)$ DoF:

$$
\mathrm{P}\left(-t_{\gamma}<\sqrt{n-1} \frac{\bar{\theta}-\theta}{\sigma}<t_{\gamma}\right)=99,9 \%
$$

The analysis of the results shows, as expected, that the first moments for axis 2 and 3 are greater than the others, because of the gravity effects. Axis 2 inertia is the largest, with greater standard deviation and confidence interval, due to its large variation with respect to robot configuration space. The inertia of the wrist links are the lower than those of the shoulder joints, as expected from their smaller size.

\subsection{Model Validation}

The model was validated comparing the real behaviour of the robot with the one simulated using the estimated parameters from Table 3; reference trajectories with trapezoidal velocity shape were used, moving one joint at time.

The error between simulated and measured data was computed together with its PDF. The position error was always lower than $0.1^{\circ}$, while the torque error was lower than $0.2 \mathrm{Nm}$.

The error PDFs were compared with gaussian ones (see Figures 8 and 9) and, apart from the first link, all others are in good accordance with gaussian distribution; therefore we can conclude that the estimated model is a valid approximation of the true system in the assumed frequency range.

\subsection{Model-Based Controller Design}

A model-based controller was designed for the 6-th joint, using the estimated robot model, in order to test the relevance of the identification procedure for control purposes, then implemented on the Linux-RTAI real-time architecture on the PC connected with the robot in our Laboratory, as described in [15]. 
Tab. 3: Estimated Robot Parameters

\begin{tabular}{|c||cr|c|c|c|}
\hline Axis & Parameter & $\bar{\theta}$ & $\sigma$ & $\frac{t_{\gamma}}{\sqrt{n-1}} \sigma$ \\
\hline \hline 1 & $\Gamma_{1}^{z z}$ & {$\left[\mathrm{kgm}^{2}\right]$} & 28,7822 & 0,3207 & 0,2179 \\
\hline \multirow{2}{*}{2} & $\Gamma_{2}^{z z}$ & {$\left[\mathrm{kgm}^{2}\right]$} & 64,1881 & 1,8838 & 1,2800 \\
& $m x_{2}$ & {$[\mathrm{kgm}]$} & 39,9920 & 0,2252 & 0,1530 \\
& $m y_{2}$ & {$[\mathrm{kgm}]$} & 6,0617 & 0,0539 & 0,0366 \\
\hline \multirow{3}{*}{3} & $\Gamma_{3}^{z z}$ & {$\left[\mathrm{kgm}^{2}\right]$} & 7,4838 & 0,1214 & 0,0825 \\
& $m x_{3}$ & {$\left[\mathrm{kgm}^{2}\right]$} & 2,8006 & 0,0115 & 0,0078 \\
& $m y_{3}$ & {$\left[\mathrm{kgm}^{2}\right]$} & $-5,6097$ & 0,0147 & 0,0100 \\
\hline \multirow{3}{*}{4} & $\Gamma_{4}^{z z}$ & {$\left[\mathrm{kgm}^{2}\right]$} & 0,2844 & 0,0107 & 0,0072 \\
& $m x_{4}$ & {$\left[\mathrm{kgm}^{2}\right]$} & $-0,0257$ & 0,0035 & 0,0024 \\
& $m y_{4}$ & {$\left[\mathrm{kgm}^{2}\right]$} & 0,0063 & 0,0048 & 0,0033 \\
\hline \multirow{3}{*}{5} & $\Gamma_{5}^{z z}$ & {$\left[\mathrm{kgm}^{2}\right]$} & 0,3541 & 0,0099 & 0,0067 \\
& $m x_{5}$ & {$\left[\mathrm{kgm}^{2}\right]$} & $-0,0518$ & 0,0030 & 0,0020 \\
& $m y_{5}$ & {$[\mathrm{kgm}]$} & 0,1428 & 0,0028 & 0,0019 \\
\hline \multirow{2}{*}{6} & $\Gamma_{6}^{z z}$ & {$\left[\mathrm{kgm}^{2}\right]$} & 0,4601 & 0,0436 & 0,0296 \\
& $m x_{6}$ & {$[\mathrm{kgm}]$} & 0,0313 & 0,0152 & 0,0103 \\
& $m y_{6}$ & {$\left[\mathrm{kgm}^{2}\right]$} & $-0,0194$ & 0,0169 & 0,0115 \\
\hline
\end{tabular}

In Figure 10 the track error was reported, relative to two different controllers, i.e. the original controller used during the identification process (a simple PID-type control) and that based on the identified model. After the initial transient, a reduction of the maximum error peak occurs when there is a change of sign in the velocity; furthermore the model-based controller is faster than original.

We are aware that these are only preliminary results and the controller needs still to be tested on the entire structure and tuned to improve its performances.

\section{Conclusion}

The aim of this work was to identify the model of an industrial robot and use it in model-based (computed torque) controller design. A rigid structure was assumed, so its range of validity is limited to low frequencies (approximately under $1 \mathrm{~Hz}$ ). A periodic exciting trajectory with a band-limited frequency contents was used, in order to compute joints velocities and accelerations analytically. A serious obstacle for a correct estimation was the presence of the original controller in the feedback loop. Neglecting its presence could lead biased or unreasonable parameters; to avoid this fact we choose to use the Projection Method, that is valid choice for closed loop systems.

The identification procedure was applied to the COMAU Smart-3 S2 robot moving one joint at time. The experimental result showed that the presented approach is feasible, and we obtained a model that afterward was successively used for controller design.

To further improve the results, the control validation shall be extended to all links, flexible modes of the structure identified and a more accurate model of the friction torques introduced.

\section{References}

[1] B. Bona, Modellistica dei Robot Industriali, Celid, 2002.

[2] K. Kozlowzki, Modelling and Identification in Robotics, Springer, 1998.

[3] M. Gautier and W. Khalil, A Direct Determination of Minimum Inertial Parameter of Robot, Proceedings of the 

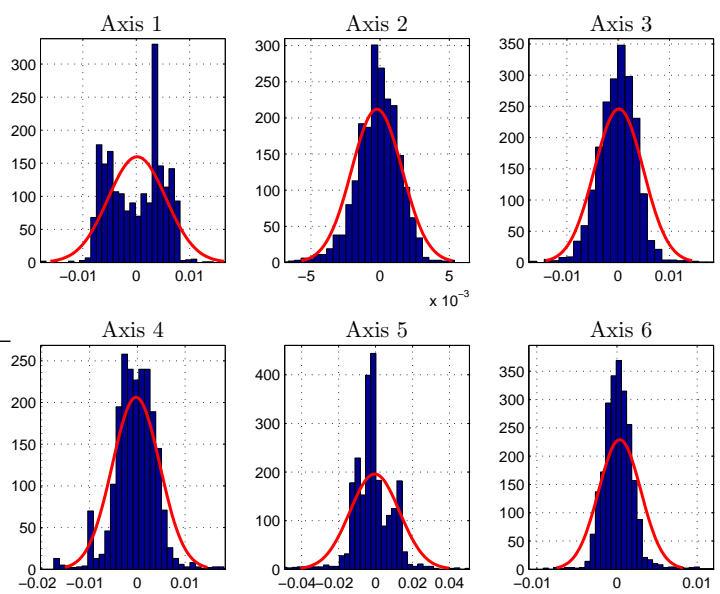

Fig. 8: Position Simulation Error PDF
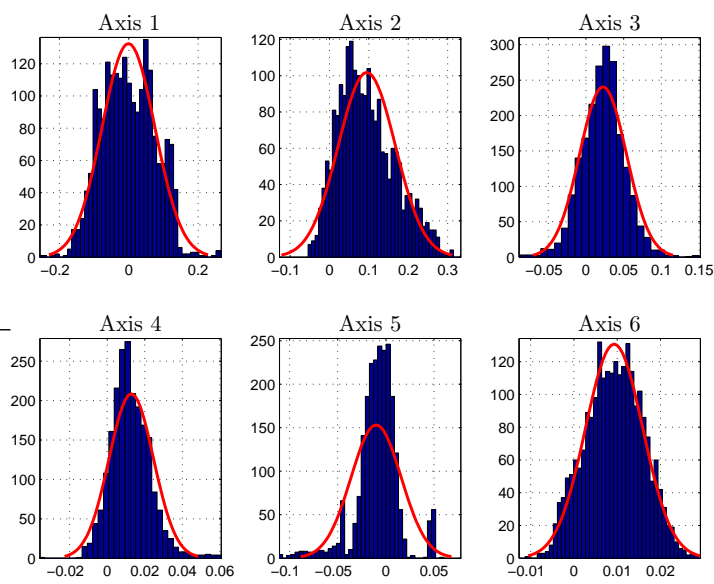

Fig. 9: Torque Simulation Error PDF

IEEE International Conference on Robotics and Automation, Philadelphia, pp. 1682-1686, 1988.

[4] M. Gautier and W. Khalil, Direct Calculation of Minimum Set of Inertial Parameters of Serial Robots, IEEE Transactions on Robotics and Automation, vol. 6, pp. 368-373, June 1990.

[5] H. Mayeda, K. Yoshida and K. Osuka, Base Parameter of Manipulator Dynamic Models, IEEE Transactions on Robotics and Automation, vol. 6(3), pp. 312-321, June 1990.

[6] C. M. Pham, M. Gautier, Essential Parameters of Robots, Proceedings of the 30th Conference on Decision and Control, Bristol, England, pp. 2769-2774, December 1991.

[7] L. Ljung, System Identification: Theory for the User, Englewood Cliffs, NJ: Prentice-Hall, 1987. 


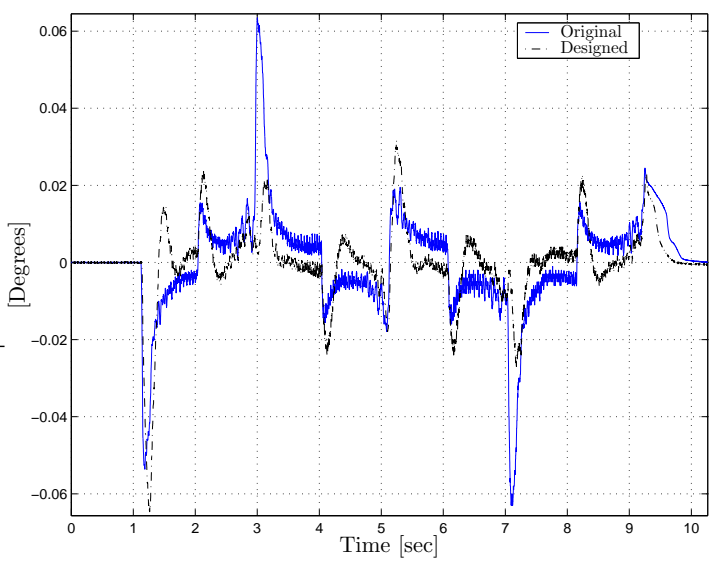

Fig. 10: Controller Track Error Axis 6

[8] U. Forssell Closed-Loop Identification: Methods, Theory and Application, Linköping University, Sweden, 1999.

[9] U. Forssell and L. Ljung A Projection Method for Closed-Loop Identification, IEEE Transactions on Automatic Control, Vol. 45(11), pp. 2101-2106, November 2000.

[10] L. Sun, H. Ohmori and A. Sano Direct Closed-Loop Identification Approach to Unstable Plant, Proceedings of the $39^{\text {th }}$ IEEE Conference on Decision and Control, Sydney, December 2000.

[11] J. S. Welsh and G. C. Goodwin Finite Sample Proprieries of Indirect Non Parametric Closed-Loop Identification, IEEE Transactions on Automatic Control, Vol. 47(8), August 2002.

[12] M.M. Olsen, Parameter Estimation of Robot Models, Ph.D. Thesis, The Maersk Mc-Kinney Moller Institute for Production Technology, University of Southern Denmark, January 2003.

[13] J. Swevers, C. Ganseman, D. B. Tükel, J. De Schutter, H. Van Brussel, Optimal Robot Excitation and Identification, IEEE Transactions on Robotics and Automation, Vol.13(5), pp. 730-740, October 1997.

[14] C. Presse and M. Gautier, New Criteria of Exciting Trajectories for Robot Identification, Proceedings of the IEEE International Conference on Robotics and Automation, pp. 907-912, Atlanta, 1993.

[15] G. Alotto, B. Bona, T. Calvelli, Prototyping Advanced Real-Time Robotic Controllers on Linux RTAI Systems with Automatic Code Generation, Proceeding of the IEEE Conference on Mechatronics and Robotics 2004, Aachen, September 2004. 